Published in final edited form as:

Lancet Oncol. 2014 January ; 15(1): 69-77. doi:10.1016/S1470-2045(13)70551-5.

\title{
Safety and Activity of PD1 Blockade by Pidilizumab in Combination with Rituximab in Patients with Relapsed Follicular Lymphoma: a Single Group, Open-label, Phase 2 Trial
}

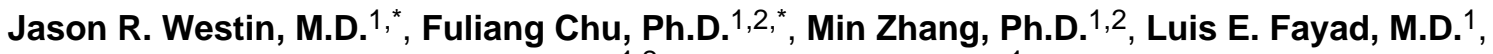 \\ Professor Larry W. Kwak, M.D., Ph.D. ${ }^{1,2}$, Nathan Fowler, M.D. ${ }^{1}$, Professor Jorge \\ Romaguera, M.D. ${ }^{1}$, Professor Fredrick Hagemeister, M.D. ${ }^{1}$, Michelle Fanale, M.D. ${ }^{1}$, Felipe \\ Samaniego, M.D. ${ }^{1}$, Lei Feng, M.S. ${ }^{3}$, Veerabhadran Baladandayuthapani, Ph.D. ${ }^{3}$, Zhiqiang \\ Wang, Ph.D. ${ }^{1,2}$, Wencai Ma, Ph.D. ${ }^{1,2}$, Yanli Gao, M.S. ${ }^{1}$, Professor Michael Wallace, M.D. ${ }^{4}$, \\ Luis M. Vence, Ph.D. ${ }^{2,5}$, Professor Laszlo Radvanyi, Ph.D. ${ }^{2,5}$, Tariq Muzzafar, M.D. ${ }^{6}$, Rinat \\ Rotem-Yehudar, Ph.D. ${ }^{7}$, R. Eric Davis, M.D. ${ }^{1,2,{ }^{* \star}}$, and Sattva S. Neelapu, M.D. ${ }^{1,2,{ }^{* *}}$ \\ ${ }^{1}$ Department of Lymphoma and Myeloma, The University of Texas M. D. Anderson Cancer \\ Center, Houston, TX \\ ${ }^{2}$ Center for Cancer Immunology Research, The University of Texas M. D. Anderson Cancer \\ Center, Houston, TX \\ ${ }^{3}$ Department of Biostatistics, The University of Texas M. D. Anderson Cancer Center, Houston, \\ TX \\ ${ }^{4}$ Department of Interventional Radiology, The University of Texas M. D. Anderson Cancer Center, \\ Houston, TX \\ ${ }^{5}$ Department of Melanoma Medical Oncology, The University of Texas M. D. Anderson Cancer \\ Center, Houston, TX \\ ${ }^{6}$ Department of Hematopathology, The University of Texas M. D. Anderson Cancer Center, \\ Houston, TX \\ ${ }^{7}$ Cure Tech Ltd., Yavne, Israel
}

\begin{abstract}
Background-Endogenous or iatrogenic antitumor immune responses can improve the course of follicular lymphoma (FL), but may be diminished by immune checkpoints in the tumor microenvironment. These may include effects of programmed death (PD)-1, a co-inhibitory receptor that impairs T-cell function and is highly expressed on intratumoral T cells. In a Phase II trial, we determined the activity of pidilizumab, a humanized anti-PD-1 monoclonal antibody, with rituximab in patients with relapsed FL.
\end{abstract}

\footnotetext{
Correspondence: Sattva S. Neelapu, M.D., 1515 Holcombe Blvd, Unit 903, The University of Texas MD Anderson Cancer Center,

Houston, TX 77030, Telephone: (713) 563 3429, Fax: (713) 563 3469, sneelapu@ @danderson.org.

JW and FC contributed equally to this work.

** RED and SSN contributed equally to this work.

Contributors

SSN and RRY designed the study. JRW, LEF, LWK, NF, JR, FH, MF, FS, and SSN participated in patient enrollment, treatment of patients, data collection, data analysis, and data interpretation. FC, MZ, ZW, WM, YG, LMV, LR, TM, RED, and SSN participated in performing correlative studies, data analysis, and data interpretation. LF and VB performed statistical analysis. MW obtained tumor biopsies for correlative studies. JRW, FC, RED, and SSN wrote the manuscript. All authors read and approved the final manuscript.

Conflicts of interest

SSN received research support from Cure Tech Ltd, Yavne, Israel. RRY is an employee of Cure Tech Ltd.
} 
Methods-FL patients with rituximab-sensitive disease relapsing after 1-4 prior therapies were eligible. Pidilizumab was administered at $3 \mathrm{mg} / \mathrm{kg}$ every 4 weeks for 4 infusions, plus 8 optional infusions every 4 weeks for patients with stable disease or better. Starting 2 weeks after the first infusion of pidilizumab, rituximab was given at $375 \mathrm{mg} / \mathrm{m}^{2}$ weekly for 4 weeks. The primary endpoint was to assess the overall response rate. Analysis was by intention to treat. Peripheral blood and tumor biopsies were studied to assess immunological effects of pidilizumab. This trial has been completed and was registered at www.clinicaltrials.gov as NCT00904722.

Findings-The combination was well-tolerated, with no autoimmune or therapy-related grade $3 / 4$ toxicities. The most common grade 1 adverse events were anemia (14 patients) and fatigue (13 patients), and the most common grade 2 adverse event was respiratory infection (5 patients). Overall 19/29 (66\%) and complete 15/29 (52\%) response rates in 29 evaluable patients were high, with tumor regression in 25/29 (86\%) of patients. Median progression-free survival was 18.8 months (95\% CI: 14.7 months to not reached). The median response duration for the 19 responders was 20.2 months (95\% CI: 13.9 months to not reached). Correlative studies of blood and tumor provided insights into predicting response and understanding mechanisms involved.

Interpretation-Pidilizumab with rituximab is well-tolerated and its activity compared favorably to historical retreatment with rituximab monotherapy in patients with relapsed FL. Our results establish that immune checkpoint blockade is worthy of further study in FL.

Funding - National Institutes of Health, Leukemia and Lymphoma Society, Cure Tech Ltd, and UT MD Anderson Cancer Center.

\section{Introduction}

The natural history of follicular lymphoma (FL), the most common indolent non-Hodgkin lymphoma worldwide, is characterized by stable disease or even spontaneous remissions, lasting months to years prior to progression. ${ }^{1}$ This suggests a transition from immune surveillance and equilibrium to escape, ${ }^{2}$ and is supported by numerous studies characterizing the influence of the immune system on FL. In a landmark study, Dave and colleagues demonstrated that survival duration of patients with FL correlated with gene expression signatures of infiltrating nonmalignant immune cells. ${ }^{3}$ An immunosurveillance pattern $\left(\mathrm{CD} 8^{+} \mathrm{T}\right.$ cells) or an immune-escape pattern $\left(\mathrm{CD} 57^{+} \mathrm{T}\right.$ cells) correlated with good or poor prognosis, respectively, in other FL studies. ${ }^{4,5}$ Tumor-specific $\mathrm{T}$ cells can also be isolated from the peripheral blood (PB) and tumor microenvironment in FL.6, 7 Together, these results suggest that endogenous antitumor immune responses are naturally induced in patients with FL but eventually rendered ineffective, possibly due to immune escape or immune checkpoints in the tumor microenvironment. ${ }^{8,9}$ Blocking immune checkpoints may promote or unleash an endogenous antitumor immune response and augment the efficacy of immunotherapeutic interventions.

Programmed death (PD)-1 is an inhibitory receptor expressed by activated $\mathrm{T}$ cells, activated B cells, NK cells, and myeloid cells. PD-1 inhibits T-cell activation when engaged by its ligands PD-L1 or PD-L2, expressed on tumor cells and/or stromal cells. ${ }^{10} \mathrm{PD}-1$ is markedly upregulated on $\mathrm{CD}^{+}{ }^{+}$and $\mathrm{CD} 8^{+} \mathrm{T}$ cells after chronic antigenic stimulation by viral infection or tumor exposure. High PD-1 expression is associated with T-cell exhaustion, and blockade of the PD-1/PD-ligand pathway with antibodies against PD-L1 and/or PD-1 augmented and/ or restored the function of viral and tumor-specific $\mathrm{CD} 4^{+}$and $\mathrm{CD} 8^{+} \mathrm{T}$ cells in mouse and human studies. ${ }^{11}$ In FL patients, PD-1 is also highly expressed on intratumoral and PB $\mathrm{CD}^{+}$and $\mathrm{CD} 8^{+} \mathrm{T}$ cells, and associated with impaired T-cell function. ${ }^{12,13}$ Therefore, targeting the PD-1/PD-ligand pathway may enhance endogenous antitumor immune responses in FL. 
Pidilizumab (formerly CT-011) is a humanized IgG-1 kappa recombinant monoclonal antibody that targets PD-1. In preclinical studies, CT-011 and BAT, the mouse monoclonal antibody from which CT-011 was derived, inhibited growth of melanoma, lymphoma, lung, colon, and breast tumors and extended the survival of mice. ${ }^{14-17}$ Selective depletion of T or NK cells in tumor-bearing mice reduced the efficacy of BAT, suggesting that both T cells and NK cells are necessary for the in vivo antitumor effect of this antibody. ${ }^{15}$ In a phase I clinical trial in patients with advanced hematological malignancies, CT-011 was found to be safe and well tolerated with no observed treatment- or infusion-related serious adverse events. Evidence of activity included a patient with FL who achieved durable complete remission. ${ }^{18}$

The monoclonal antibody rituximab, directed against the B cell antigen CD20, is utilized alone and in combination to treat FL, in both the frontline and relapse setting. Rituximab has improved response rates, progression-free survival (PFS), and overall survival (OS) of patients with FL. ${ }^{19-22}$ Patients previously treated with single-agent rituximab have been successfully retreated after relapse. ${ }^{23,24}$ Rituximab acts in part via activation of NK cellmediated antibody-dependent cellular cytotoxicity (ADCC). Therefore, we reasoned that the combination of pidilizumab and rituximab would have additive and/or synergistic effects via activation of both the innate (NK cells) and adaptive (T cells) arms of the immune system, enhancing clinical efficacy without increasing toxicity. Here, we report safety, activity, and correlative studies of pidilizumab and rituximab from a single-arm phase II trial in patients with relapsed FL.

\section{Methods}

Patients

In this institutional review board-approved single-institution, open-label, nonrandomized phase II trial, grade 1-2 adult FL patients relapsing after 1-4 prior therapies with rituximabsensitive disease, defined as complete or partial response lasting $\geq 6$ months were enrolled after written informed consent in accordance with the Declaration of Helsinki. Other inclusion criteria included measurable disease, performance status $<2$, absolute neutrophil count $\geq 1.5 \times 10^{9} / \mathrm{L}$, absolute lymphocyte count (ALC) $\geq 0.6 \times 10^{9} / \mathrm{L}$, platelets $250 \times 10^{9} / \mathrm{L}$, and adequate organ function. Patients with active infection, central nervous system lymphoma, autoimmune diseases or allogeneic stem cell transplantation were excluded. This trial was registered at www.clinicaltrials.gov as NCT00904722.

\section{Treatment}

Pidilizumab was dosed at $3 \mathrm{mg} / \mathrm{kg}$ intravenously every 4 weeks for 4 infusions. Rituximab was dosed at $375 \mathrm{mg} / \mathrm{m}^{2}$ intravenously weekly for 4 weeks starting day 17 after the first infusion of pidilizumab. Patients with stable disease (SD) or better received 8 additional optional infusions of pidilizumab every 4 weeks for a total of 12 doses. Dose modifications were not permitted for pidilizumab or rituximab. Dose interruption of up to 3 weeks was permitted for pidilizumab for grade 3 or higher toxicity.

\section{Assessment of response and toxicity}

The primary objective was to determine the overall response rate (ORR). The secondary objectives were to determine the safety and toxicity, complete (CR) and partial (PR) response rates, PFS, and immunological effects of pidilizumab. Adverse events were graded according to the Common Terminology Criteria for Adverse Events version 3.0. Response was determined according to Revised Response Criteria for Malignant Lymphoma using computed tomography (CT) scans and bone marrow biopsy. ${ }^{25}$ Positron emission tomography (PET)-CT scan was performed at the discretion of the treating physician and 
was also used to assess response when performed. Assessments were performed after completion of the second and fourth infusions of pidilizumab, and every 12 weeks thereafter for 2 years or until relapse.

\section{Flow cytometric analysis}

Immunophenotyping was performed on PB mononuclear cells (PBMC) by flow cytometry prior to and on day 14 after the first infusion of pidilizumab. Data were acquired using a BD LSRFortessa $^{\text {TM }}$ Cell Analyzer (BD Biosciences) and analyzed using FlowJo v9.5.2 software (Tree Star, Inc.).

\section{Gene expression profiling (GEP)}

Core needle biopsies from involved lymph nodes, obtained before and 14 days after the first infusion of pidilizumab, were collected in RNAlater fixative and stored at $-80^{\circ} \mathrm{C}$ until RNA isolation. For samples with adequate RNA quantity and quality, determined with an Agilent 2100 Bioanalyzer, $300 \mathrm{ng}$ of total RNA was amplified and biotin-labeled by the Eberwine method for hybridization to HT-12 version 4 BeadArrays from Illumina. Data processing was performed as previously described, ${ }^{26}$ and genes were excluded if expression levels were not significantly above background in at least $25 \%$ of samples. Gene Set Enrichment Analysis (GSEA; http://www.broadinstitute.org/gsea) was performed with assigned gene ranking. To find gene signatures that correlate significantly with PFS, we used a novel method (manuscript in preparation). In brief, for each specific signature and sample, a signature score was based on the expression level of signature genes, then used in a univariate Cox proportional hazards model test of correlation with PFS. The predictive power of the signature, defined as the negative logarithm of the $p$ value from the Cox test, was then compared to a null distribution of the similarly-determined predictive power of 1000 signatures of randomly-selected genes. The significance $p$ value of the signature's predictive power was then based on its relative rank in the null distribution.

\section{Statistical analysis}

At the time of trial design, pidilizumab and rituximab combination therapy was expected to induce an ORR of $60 \%$ as compared with ORR of $40 \%$ expected with rituximab monotherapy when used as retreatment in FL patients. ${ }^{23}$ PFS was measured from enrollment to disease progression or recurrence or death from any cause. Patients were censored at the last disease evaluation if progression has not occurred. Paired student's t-test was used to determine the significance of change in biomarkers between time points. Fisher's exact test was used to evaluate the association between response status and other patient characteristics. Wilcoxon rank-sum test was used to evaluate differences in marker expression between responders and non-responders. Kaplan-Meier method was used for time-to-event analysis. The log-rank test was used to evaluate the difference in time-to-event endpoints between patient groups. Univariate Cox proportional hazards models were fitted to evaluate the correlation with PFS of biomarkers, including tumor shrinkage, and GEP signatures. Statistical software SAS 9.1.3 (SAS, Cary, NC) and S-Plus 8.0 (TIBCO Software Inc., Palo Alto, CA) were used for the analyses.

\section{Role of the funding source}

This was an investigator-initiated study. Pidilizumab was provided free of cost by Cure Tech Ltd, Yavne, Israel. National Institutes of Health, Bethesda, Maryland, USA; Leukemia and Lymphoma Society; Cure Tech Ltd; and UT MD Anderson Cancer Center, Houston, Texas, USA provided funding to conduct the study and correlative studies. The sponsors had no role in study design, data collection, data analysis, data interpretation, or writing. The 
authors (JRW, FC, RED, and SSN) had full access to all raw data and final responsibility for the decision to write and submit this for publication.

\section{Results}

Thirty-two patients were enrolled between January 13, 2010 and January 20, 2012. Two patients were ineligible and not treated, and one patient was withdrawn after one infusion of pidilizumab and received alternative treatment as per the treating physician's decision. Thus, 30 patients were evaluable for toxicity and 29 patients were eligible for efficacy analysis. Characteristics of the 30 treated patients are summarized in Table 1. Patients were fairly well distributed among the three risk groups of FL International Prognostic Index (FLIPI) $1^{27}$ and FLIPI2. ${ }^{28}$ All patients had received rituximab previously either as monotherapy or in combination and 21/30 (70\%) received combination chemotherapy or chemoimmunotherapy. The median number of prior treatments was 1 (range, 1-4).

The median number of pidilizumab infusions administered for the 30 patients was 10 (range, 1-12) and 29 patients received the four infusions of rituximab as per protocol. The treatment was well tolerated with no autoimmune or therapy-related grade 3 or 4 adverse events. No patient had dose interruption or discontinued therapy due to toxicity. Grade 1 and 2 adverse events observed in $\geq 10 \%$ of patients regardless of attribution are summarized in Table 2.

Of the 29 patients evaluable for efficacy analysis, 19 achieved an objective response for an ORR of 66\%. CR was observed in 15/29 (52\%) and PR in 4/29 patients (14\%). Follow-up PET-CT scan was performed in 9 patients and confirmed CR. Altogether, 25/29 (86\%) patients had measurable tumor regression (Figure 1a). The median time to observed response was 88 days (range, 53-392). Six/29 (21\%) patients had considerably delayed response with the initial response $>4$ months after first pidilizumab infusion (Figure 1b). The median follow-up was 15.4 months, ranging from 1.8-35.0 months (IQR 10.1-21.0 months). The median PFS for all patients was 18.8 months (95\% confidence interval (CI): 14.7, NA) (Figure 2a) but was not reached (95\% CI: 18.8, NA) for the 19 responders, and was 19.6 months (95\% CI: 17.5, NA) for the 25 patients with measurable tumor regression. The median response duration for the 19 responders was 20.2 months (95\% CI: 13.9, NA) and only seven responders progressed to date. Clinical response was not significantly associated with FLIPI1, FLIPI2, prior therapy, number of prior rituximab doses, or duration of response to prior therapy $(\mathrm{p}>0.05)$. However, PFS was significantly associated with both FLIPI1 (median PFS for low/intermediate versus high risk, not reached (95\% CI: 15.3, NA) versus 13.7 months (95\% CI: 3.7, NA; $\mathrm{p}=0.01$ ) (Figure 2b) and FLIPI2 (median PFS for low/ intermediate versus high, not reached (95\% CI: 15.3, NA) versus 14.1 months (95\% CI: 12.7, NA); $\mathrm{p}<0.01$ ) (Figure 2c). There were no deaths on the trial.

Correlative studies on baseline samples implicated factors predictive and/or explanatory of response to PD-1 blocking. Expression of PD-L1 (but not PD-1 or PD-L2), a marker of endogenous antitumor immunity, ${ }^{11,29}$ was significantly higher in $\mathrm{PB} \mathrm{CD} 4^{+}, \mathrm{CD}^{+}$, and $\mathrm{CD} 14^{+}$cells among responders compared with non-responders (Figures $3 \mathrm{a}-\mathrm{c}$ ), but was not associated with PFS ( $p>0.05$ ). GEP data of baseline tumor biopsies from 18 patients were analyzed to find multi-gene signatures correlating with PFS, after assigning to each patient a score based on the expression of signature genes. Among publicly-curated signatures significantly predictive of longer PFS in our dataset (Table 3), prominent were signatures of genes upregulated during T-cell activation, or repressed in regulatory $\mathrm{T}$ cells, often with considerable overlap, suggesting that endogenous antitumor immunity at baseline predicted better response to pidilizumab. Significant positive correlation with PFS was also observed for a signature created by GEP studies we did on $\mathrm{CD} 4{ }^{+} \mathrm{T}$ cells sorted from a separate group of banked FL tumor biopsies. In brief, we identified 41 genes more highly expressed in 
effector $\mathrm{T}$ cells $\left(\mathrm{T}_{\text {effs }}\right.$, PD- $\left.1^{\text {int }} \mathrm{CXCR} 5^{\text {int }} / \mathrm{PD}-1^{\mathrm{lo}} \mathrm{CXCR} 5^{\mathrm{lo}}\right)$, as compared to follicular helper $\mathrm{T}$ cells $\left(\mathrm{T}_{\mathrm{FH}}, \mathrm{PD}-1^{\text {hi }}\right.$ CXCR5 ${ }^{\mathrm{hi}}$ ) (Supplementary Figures $1 \mathrm{a}$ and $1 \mathrm{~b}$ ). Consistent with our expectation that $\mathrm{T}_{\text {effs }}$ are likely to have antitumor effects, whereas $\mathrm{T}_{\mathrm{FH}}$ are likely to have protumor effects, ${ }^{30,31}$ we found that low expression of this signature, suggesting more $\mathrm{T}_{\mathrm{FH}}$ and fewer PD- $1^{+} \mathrm{T}_{\text {effs }}$ within the tumor, predicted less tumor shrinkage and shorter PFS: median of 12.7 months $(95 \%$ CI: 6.5, 21.6) for signature-low patients vs. not reached $(95 \%$ CI: NA, NA) for signature-high patients (Supplementary Figures 2a-c). In contrast, dichotomization by this signature did not show a significant difference in OS in an external dataset of $191 \mathrm{FL}$ patients treated largely with chemotherapy alone ${ }^{3}$ (Supplementary Figures $2 \mathrm{~d}$ and $2 \mathrm{e}$ ). This difference suggests that the predictive power of the 41-gene signature, and the process that it represents, may require the therapeutic context of anti-PD-1 antibody and/ or rituximab in order to be relevant, and not be features of the "natural history" of FL. However, this implication should be regarded as highly speculative, in need of much more documentation.

We also examined the effects of pidilizumab by comparing samples taken 14 days after the first pidilizumab infusion to baseline samples. In PB samples, there were significant increases in $\mathrm{ALC}$, and $\mathrm{CD}^{+}$and $\mathrm{CD} 4^{+} \mathrm{T}$ cells, but not in $\mathrm{CD}^{+} \mathrm{T}$ cells (Supplementary Figures $3 \mathrm{a}$ and $\mathrm{b}$ ). Naïve, effector memory, and central memory $\mathrm{CD} 4^{+} \mathrm{T}$ cells were significantly increased post-treatment (Supplementary Figure 3c). Among CD8 ${ }^{+} \mathrm{T}$-cells, terminally differentiated cells were decreased but other subsets were not significantly altered (Supplementary Figure 3d). Expression of the activating receptor NKG2D on NK cells was significantly increased ( $\mathrm{p}<0.05$; data not shown). To analyze GEP data from paired core needle biopsies from 8 patients, the change in expression after treatment was correlated with outcome. Increased expression of T-cell activation signatures (Table 4) after pidilizumab treatment was associated with longer PFS, suggesting that endogenous antitumor immune responses were enhanced by pidilizumab. We also performed GSEA, based on ranking all genes by the slope of Pearson's correlation between the pidilizumab-induced change in expression and the FTC of these 8 patients. Signatures of processes significantly associated with favorable FTC were related to $\mathrm{CD} 4^{+}$and $\mathrm{CD} 8^{+} \mathrm{T}$-cell activation, proliferation or to changes in mitochondria, particularly in genes involved in oxidative phosphorylation; these are processes upregulated in $\mathrm{T}$ cells during an acute immune response, ${ }^{32}$ again suggesting that favorable responses to pidilizumab were due to enhanced T-cell immunity (Supplementary Figures 4a-d).

\section{Discussion}

The combination of pidilizumab and rituximab was active and well-tolerated in patients with relapsed FL. The ORR (66\%) and CR rate (52\%) compare favorably with the previouslyreported ORR (40\%) and CR rate (11\%) in patients retreated with rituximab monotherapy. ${ }^{23}$ The median PFS for responders in this trial (not reached) also compares favorably with the estimated median time to progression of 17.8 months reported with rituximab monotherapy retreatment in the Davis et al and other studies. ${ }^{23,33}$ These encouraging clinical results, together with the extremely low toxicity profile of this combination, make it especially appealing for FL patients, most of whom are elderly. Although selection bias or disease assessment methods may influence the results of single arm trials, we think this is unlikely as our patient characteristics (Table 1) are typical of patients with relapsed FL and our disease assessment methods followed international standards. However, a randomized study is necessary to definitively compare the efficacy of this combination relative to rituximab monotherapy. Pidilizumab administration after autologous stem cell transplantation in diffuse large B-cell lymphoma patients was also safe and showed potential clinical benefit. ${ }^{34}$ The absence of autoimmune adverse events in our study stands in contrast to the immunerelated adverse events reported with other anti-PD-1 antibodies, nivolumab and 
lambrolizumab. ${ }^{29,35}$ This difference may be due to the higher dose and more frequent administration used for nivolumab and lambrolizumab; prior exposure to ipilimumab, an anti-CTLA-4 antibody associated with autoimmune adverse events, in the nivolumab and lambrolizumab studies; B-cell depletion induced by rituximab in our study; and possibly a more immunocompromised state of FL patients. Randomized studies are needed to directly compare both safety and efficacy of these anti-PD-1 antibodies. Furthermore, the safety and efficacy of long-term therapy with pidilizumab with and without rituximab maintenance needs to be explored.

Therapeutic agents that target immune checkpoints are expected to enhance endogenous antitumor immune responses and therefore, benefit patients with preexisting antitumor immunity. ${ }^{11}$ Consistent with this notion, longer PFS was observed in patients with higher levels of T-cell activation signatures, and/or the 41-gene $\mathrm{T}_{\text {eff }} \mathrm{vs}$. $\mathrm{T}_{\mathrm{FH}}$ signature, in their tumors at baseline (Table 3 and Supplementary Figure 2). Since the $T_{\text {eff }}$ signature did not correlate with OS in an external dataset of FL patients treated largely with chemotherapy alone, this signature may be specific for predicting outcome after anti-PD-1 antibody therapy. However, evaluation of this signature in additional external datasets of FL patients treated with rituximab alone or confirmation in randomized studies is necessary to definitively make this association. Another marker of preexisting antitumor immunity is PD$\mathrm{L} 1$, due to the phenomenon of adaptive resistance. ${ }^{11,29,36}$ In concordance with this, responders expressed higher levels of PD-L1 on PB T cells and monocytes at baseline relative to non-responders. Moreover, comparison of baseline to day 14 samples showed that pidilizumab increased expression of activation-associated genes by T and NK cells in the PB and/or the tumor microenvironment, signatures of processes associated with T-cell immune responses, and absolute numbers of effector and memory $\mathrm{CD} 4^{+} \mathrm{T}$ cells in the $\mathrm{PB}$, suggesting that pidilizumab enhanced endogenous antitumor immune responses. Due to the small sample size, the results of our correlative studies need to be interpreted with caution and should be tested in larger studies. In addition, the results of our GEP studies need to be confirmed by flow cytometry and immunohistochemistry studies of tumor samples. Inability to perform these studies due to inadequate samples and inability to compare these results to blood and tissue samples at initial diagnosis are potential limitations of this study.

Nevertheless, multiple predictors associated with clinical outcome from pre-treatment samples, and multiple effects observed in post-treatment samples, were consistent with the expected mechanism of action of pidilizumab.

Although our analysis suggested that signatures of T-cell activation and/or $\mathrm{T}_{\text {effs }}$ at baseline are associated with PFS, the FL tumor microenvironment has multiple T-cell subsets that express PD-1: antitumor $\mathrm{T}_{\text {effs }}$ such as $\mathrm{CD} 4^{+} \mathrm{T}$ helper 1 and cytotoxic $\mathrm{CD} 8^{+} \mathrm{T}$ cells; protumor $\mathrm{T}_{\mathrm{FH}}$ : and subsets of regulatory $\mathrm{T}$ cells, including recently-described follicular regulatory $\mathrm{T}$ cells $\left(\mathrm{T}_{\mathrm{FR}}\right)$ that may suppress tumor $\mathrm{B}$ cells and $\mathrm{T}_{\mathrm{FH}} \cdot{ }^{12,13,30,31,37}$

Inconsistency of reports on the prognostic effect of PD- $1^{+}$lymphocytes in FL as assessed by immunohistochemistry ${ }^{38-42}$ might therefore be due to the multiplicity of PD- $1^{+}$T-cell subsets. While PD-1 blockade enhances the function of antitumor $\mathrm{T}_{\mathrm{effs}}, 10,11$ its effects on other PD- $1^{+}$T-cell subsets are unclear. Future studies of anti-PD-1 antibody in FL should incorporate strategies to enumerate these subsets in tumor samples, as it is likely that the net effect of PD-1 blockade may depend on the relative proportion of the various PD- $1^{+}$T-cell subsets.

Animal and human studies suggest that PD- $1^{+} \mathrm{T}$ cells may also express other inhibitory receptors such as CTLA-4, TIM-3, LAG-3, BTLA, CD160, CD244, and others. 11, 43, 44 Therefore, blocking PD-1 alone may not fully restore the function of antitumor T cells. Indeed, CTLA-4 and TIM-3 are expressed on intratumoral T cells in FL and ipilimumab therapy has been associated with clinical responses in FL. ${ }^{8,12,45-47}$ Thus, combining 
pidilizumab with other immune checkpoint inhibitors ${ }^{48}$ may further enhance the endogenous antitumor T-cell responses and improve clinical outcome in these patients. Combining pidilizumab with immunostimulatory agents such as vaccines, ${ }^{49}$ Toll-like receptor ligands, ${ }^{50}$ lenalidomide and/or agonists of OX-40 51 and $4-1 \mathrm{BB}^{52}$ may also be a rational approach.

In summary, our results suggest that pidilizumab and rituximab therapy is active and welltolerated in patients with relapsed, rituximab-sensitive FL. Pidilizumab appears to activate T and NK cells and enhance endogenous antitumor immune responses. Further evaluation of pidilizumab with rituximab and/or other immunomodulatory agents is warranted.

\section{Supplementary Material}

Refer to Web version on PubMed Central for supplementary material.

\section{Acknowledgments}

This work was supported by grants from the National Institutes of Health R21 CA143785 (SSN) and R01 CA155143 (SSN and RED), Leukemia and Lymphoma Society Specialized Center of Research grant 7262-08 (SSN, LMV, LR, and LWK), the University of Texas MD Anderson Cancer Center, and Cure Tech Ltd. This work was also supported by the NIH Clinical and Translational Science Award UL1 RR024148 and by the NIH Cancer Center Support Grant (CCSG) award CA16672 to MD Anderson Cancer Center (LF and VB).

\section{References}

1. Horning SJ, Rosenberg SA. The Natural History of Initially Untreated Low-Grade Non-Hodgkin's Lymphomas. New England Journal of Medicine. 1984; 311(23):1471-5. [PubMed: 6548796]

2. Dunn GP, Old LJ, Schreiber RD. The three Es of cancer immunoediting. Annu Rev Immunol. 2004; 22:329-60. [PubMed: 15032581]

3. Dave SS, Wright G, Tan B, et al. Prediction of survival in follicular lymphoma based on molecular features of tumor-infiltrating immune cells. N Engl J Med. 2004; 351(21):2159-69. [PubMed: 15548776]

4. Wahlin BE, Sander B, Christensson B, Kimby E. CD8+ T-cell content in diagnostic lymph nodes measured by flow cytometry is a predictor of survival in follicular lymphoma. Clin Cancer Res. 2007; 13(2 Pt 1):388-97. [PubMed: 17255259]

5. Alvaro T, Lejeune M, Salvado MT, et al. Immunohistochemical patterns of reactive microenvironment are associated with clinicobiologic behavior in follicular lymphoma patients. $\mathrm{J}$ Clin Oncol. 2006; 24(34):5350-7. [PubMed: 17135637]

6. Schultze JL, Seamon MJ, Michalak S, Gribben JG, Nadler LM. Autologous tumor infiltrating T cells cytotoxic for follicular lymphoma cells can be expanded in vitro. Blood. 1997; 89(10):380616. [PubMed: 9160688]

7. Lee ST, Liu S, Radvanyi L, et al. A novel strategy for rapid and efficient isolation of human tumorspecific CD4(+) and CD8(+) T-cell clones. J Immunol Methods. 2008; 331(1-2):13-26. [PubMed: 17959194]

8. Yang ZZ, Ansell SM. The tumor microenvironment in follicular lymphoma. Clin Adv Hematol Oncol. 2012; 10(12):810-8. [PubMed: 23271353]

9. Kridel R, Sehn LH, Gascoyne RD. Pathogenesis of follicular lymphoma. J Clin Invest. 2012; 122(10):3424-31. [PubMed: 23023713]

10. Keir ME, Butte MJ, Freeman GJ, Sharpe AH. PD-1 and its ligands in tolerance and immunity. Annu Rev Immunol. 2008; 26:677-704. [PubMed: 18173375]

11. Pardoll DM. The blockade of immune checkpoints in cancer immunotherapy. Nat Rev Cancer. 2012; 12(4):252-64. [PubMed: 22437870]

12. Yang ZZ, Novak AJ, Stenson MJ, Witzig TE, Ansell SM. Intratumoral CD4+CD25+ regulatory Tcell-mediated suppression of infiltrating CD4+ T cells in B-cell non-Hodgkin lymphoma. Blood. 2006; 107(9):3639-46. [PubMed: 16403912] 
13. Myklebust JH, Irish JM, Brody J, et al. High PD-1 expression and suppressed cytokine signaling distinguish T cells infiltrating follicular lymphoma tumors from peripheral T cells. Blood. 2013; 121(8):1367-76. [PubMed: 23297127]

14. Hardy B, Indjiia L, Rodionov G, Raiter A, Inbal A. Treatment with BAT monoclonal antibody decreases tumor burden in a murine model of leukemia/lymphoma. Int J Oncol. 2001; 19(5):897902. [PubMed: 11604985]

15. Hardy B, Niv Y, Fadaeev L, Raiter A. BAT mAb induces lymphopoiesis in nude mice. Int Immunol. 2005; 17(5):615-9. [PubMed: 15802304]

16. Feinmesser M, Raiter A, Hardy B. Prevention of melanoma metastases in lungs of BAT treated and peptide immunized mice. Int J Oncol. 2006; 29(4):911-7. [PubMed: 16964387]

17. Hardy B, Morgenstern S, Raiter A, Rodionov G, Fadaeev L, Niv Y. BAT monoclonal antibody immunotherapy of human metastatic colorectal carcinoma in mice. Cancer Lett. 2005; 229(2): 217-22. [PubMed: 16122870]

18. Berger R, Rotem-Yehudar R, Slama G, et al. Phase I safety and pharmacokinetic study of CT-011, a humanized antibody interacting with PD-1, in patients with advanced hematologic malignancies. Clin Cancer Res. 2008; 14(10):3044-51. [PubMed: 18483370]

19. Marcus R, Imrie K, Solal-Celigny P, et al. Phase III study of R-CVP compared with cyclophosphamide, vincristine, and prednisone alone in patients with previously untreated advanced follicular lymphoma. J Clin Oncol. 2008; 26(28):4579-86. [PubMed: 18662969]

20. Cheson BD, Leonard JP. Monoclonal antibody therapy for B-cell non-Hodgkin's lymphoma. N Engl J Med. 2008; 359(6):613-26. [PubMed: 18687642]

21. Hiddemann W, Kneba M, Dreyling M, et al. Frontline therapy with rituximab added to the combination of cyclophosphamide, doxorubicin, vincristine, and prednisone (CHOP) significantly improves the outcome for patients with advanced-stage follicular lymphoma compared with therapy with CHOP alone: Results of a prospective randomized study of the German Low-Grade Lymphoma Study Group. Blood. 2005; 106(12):3725-32. [PubMed: 16123223]

22. Fisher RI, LeBlanc M, Press OW, Maloney DG, Unger JM, Miller TP. New treatment options have changed the survival of patients with follicular lymphoma. J Clin Oncol. 2005; 23(33):8447-52. [PubMed: 16230674]

23. Davis TA, Grillo-Lopez AJ, White CA, et al. Rituximab anti-CD20 monoclonal antibody therapy in non-Hodgkin's lymphoma: safety and efficacy of re-treatment. J Clin Oncol. 2000; 18(17): 3135-43. [PubMed: 10963642]

24. Hainsworth JD, Litchy S, Shaffer DW, Lackey VL, Grimaldi M, Greco FA. Maximizing therapeutic benefit of rituximab: maintenance therapy versus re-treatment at progression in patients with indolent non-Hodgkin's lymphoma--a randomized phase II trial of the Minnie Pearl Cancer Research Network. J Clin Oncol. 2005; 23(6):1088-95. [PubMed: 15657401]

25. Cheson BD, Pfistner B, Juweid ME, et al. Revised Response Criteria for Malignant Lymphoma. Journal of Clinical Oncology. 2007; 25(5):579-86. [PubMed: 17242396]

26. Ma W, Wang M, Wang ZQ, et al. Effect of long-term storage in TRIzol on microarray-based gene expression profiling. Cancer Epidemiol Biomarkers Prev. 2010; 19(10):2445-52. [PubMed: 20805315]

27. Solal-Celigny $\mathrm{P}$, Roy $\mathrm{P}$, Colombat $\mathrm{P}$, et al. Follicular lymphoma international prognostic index. Blood. 2004; 104(5):1258-65. [PubMed: 15126323]

28. Federico M, Bellei M, Marcheselli L, et al. Follicular lymphoma international prognostic index 2: a new prognostic index for follicular lymphoma developed by the international follicular lymphoma prognostic factor project. J Clin Oncol. 2009; 27(27):4555-62. [PubMed: 19652063]

29. Topalian SL, Hodi FS, Brahmer JR, et al. Safety, activity, and immune correlates of anti-PD-1 antibody in cancer. N Engl J Med. 2012; 366(26):2443-54. [PubMed: 22658127]

30. Ame-Thomas P, Le Priol J, Yssel H, et al. Characterization of intratumoral follicular helper T cells in follicular lymphoma: role in the survival of malignant B cells. Leukemia. 2012; 26(5):1053-63. [PubMed: 22015774]

31. Rawal S, Chu F, Zhang M, et al. Cross talk between follicular Th cells and tumor cells in human follicular lymphoma promotes immune evasion in the tumor microenvironment. J Immunol. 2013; 190(12):6681-93. [PubMed: 23686488] 
32. Chang CH, Curtis JD, Maggi LB Jr, et al. Posttranscriptional control of T cell effector function by aerobic glycolysis. Cell. 2013; 153(6):1239-51. [PubMed: 23746840]

33. Coiffier B, Osmanov EA, Hong X, et al. Bortezomib plus rituximab versus rituximab alone in patients with relapsed, rituximab-naive or rituximab-sensitive, follicular lymphoma: a randomised phase 3 trial. Lancet Oncol. 2011; 12(8):773-84. [PubMed: 21724462]

34. Armand P, Nagler A, Weller EA, et al. Disabling Immune Tolerance by Programmed Death-1 Blockade With Pidilizumab After Autologous Hematopoietic Stem-Cell Transplantation for Diffuse Large B-Cell Lymphoma: Results of an International Phase II Trial. J Clin Oncol. 2013

35. Hamid O, Robert C, Daud A, et al. Safety and tumor responses with lambrolizumab (anti-PD-1) in melanoma. N Engl J Med. 2013; 369(2):134-44. [PubMed: 23724846]

36. Taube JM, Anders RA, Young GD, et al. Colocalization of inflammatory response with B7-h1 expression in human melanocytic lesions supports an adaptive resistance mechanism of immune escape. Sci Transl Med. 2012; 4(127):127ra37.

37. Chung Y, Tanaka S, Chu F, et al. Follicular regulatory T cells expressing Foxp3 and Bcl-6 suppress germinal center reactions. Nat Med. 2011; 17(8):983-8. [PubMed: 21785430]

38. Carreras J, Lopez-Guillermo A, Roncador G, et al. High numbers of tumor-infiltrating programmed cell death 1-positive regulatory lymphocytes are associated with improved overall survival in follicular lymphoma. J Clin Oncol. 2009; 27(9):1470-6. [PubMed: 19224853]

39. Wahlin BE, Aggarwal M, Montes-Moreno S, et al. A unifying microenvironment model in follicular lymphoma: outcome is predicted by programmed death-1--positive, regulatory, cytotoxic, and helper T cells and macrophages. Clin Cancer Res. 2010; 16(2):637-50. [PubMed: 20068089]

40. Richendollar BG, Pohlman B, Elson P, Hsi ED. Follicular programmed death 1-positive lymphocytes in the tumor microenvironment are an independent prognostic factor in follicular lymphoma. Hum Pathol. 2011; 42(4):552-7. [PubMed: 21237493]

41. Takahashi H, Tomita N, Sakata S, et al. Prognostic significance of programmed cell death-1positive cells in follicular lymphoma patients may alter in the rituximab era. Eur J Haematol. 2013; 90(4):286-90. [PubMed: 23331211]

42. Koch K, Hoster E, Unterhalt M, et al. The composition of the microenvironment in follicular lymphoma is associated with the stage of the disease. Hum Pathol. 2012; 43(12):2274-81. [PubMed: 22795355]

43. Blackburn SD, Shin H, Haining WN, et al. Coregulation of CD8+ T cell exhaustion by multiple inhibitory receptors during chronic viral infection. Nat Immunol. 2009; 10(1):29-37. [PubMed: 19043418]

44. Wherry EJ. T cell exhaustion. Nat Immunol. 2011; 12(6):492-9. [PubMed: 21739672]

45. Ansell SM, Hurvitz SA, Koenig PA, et al. Phase I study of ipilimumab, an anti-CTLA-4 monoclonal antibody, in patients with relapsed and refractory B-cell non-Hodgkin lymphoma. Clin Cancer Res. 2009; 15(20):6446-53. [PubMed: 19808874]

46. Yang ZZ, Grote DM, Ziesmer SC, et al. IL-12 upregulates TIM-3 expression and induces T cell exhaustion in patients with follicular B cell non-Hodgkin lymphoma. J Clin Invest. 2012; 122(4): 1271-82. [PubMed: 22426209]

47. O'Mahony D, Morris JC, Quinn C, et al. A pilot study of CTLA-4 blockade after cancer vaccine failure in patients with advanced malignancy. Clin Cancer Res. 2007; 13(3):958-64. [PubMed: 17289891]

48. Wolchok JD, Kluger H, Callahan MK, et al. Nivolumab plus ipilimumab in advanced melanoma. N Engl J Med. 2013; 369(2):122-33. [PubMed: 23724867]

49. Schuster SJ, Neelapu SS, Gause BL, et al. Vaccination with patient-specific tumor-derived antigen in first remission improves disease-free survival in follicular lymphoma. J Clin Oncol. 2011; 29(20):2787-94. [PubMed: 21632504]

50. Houot R, Levy R. T-cell modulation combined with intratumoral CpG cures lymphoma in a mouse model without the need for chemotherapy. Blood. 2009; 113(15):3546-52. [PubMed: 18941113]

51. Marabelle A, Kohrt H, Sagiv-Barfi I, et al. Depleting tumor-specific Tregs at a single site eradicates disseminated tumors. J Clin Invest. 2013; 123(6):2447-63. [PubMed: 23728179] 
52. Houot R, Goldstein MJ, Kohrt HE, et al. Therapeutic effect of CD137 immunomodulation in lymphoma and its enhancement by Treg depletion. Blood. 2009; 114(16):3431-8. [PubMed: 19641184] 


\section{Research in context}

\section{Systematic review}

The design of our study was informed by strong evidence that immunotherapy could induce meaningful and durable clinical remissions in FL. The critical interaction between the intact immune system and FL, and the role of modulating this interaction, is summarized in published works cited in the Introduction and Discussion. We did a comprehensive scientific literature search consisting of structured searches of PubMed when writing our report in July, 2013. We placed no date or language restrictions on the searches. We did four specific searches: (1) ("follicular lymphoma" AND "immune"), (2) ("follicular lymphoma" AND "rituximab" AND "immune"), (3) ("follicular lymphoma" AND "PD-1"), and (4) ("follicular lymphoma" AND "rituximab" AND "PD-1"). We did not identify any other clinical trials targeting PD-1 combined with rituximab, although several papers with in vitro data suggested the concept to be worthy of exploration.

\section{Interpretation}

Our findings suggest that the combination of rituximab and pidilizumab is an active and well-tolerated therapy, and establish that immune checkpoint blockade is worthy of further study in FL. These results also support further investigation of pidilizumab combined with other treatments, perhaps chemotherapy or additional immunemodulating therapies. 


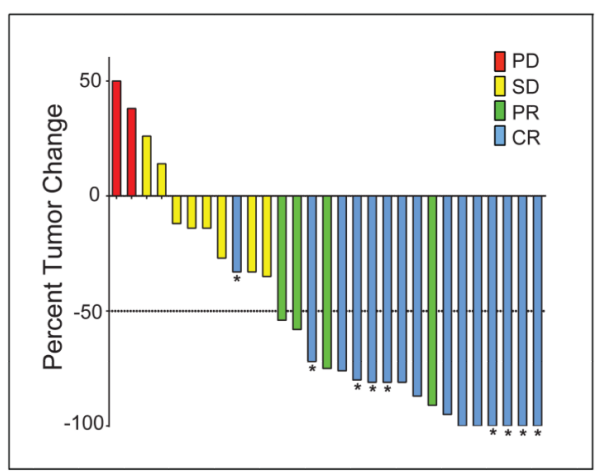

b

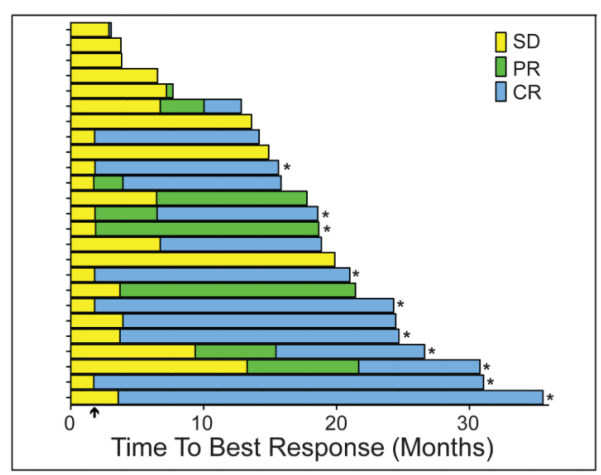

Figure 1. Clinical response after pidilizumab and rituximab therapy

a) Best response after pidilizumab and rituximab therapy. Percent change in tumors size from baseline was determined by measuring the sum of the product of the diameters of up to six tumors on CT scans. Asterisk (*) indicates patients whose complete response (CR) was confirmed by PET-CT scan. PR, partial response; SD, stable disease; PD, progressive disease. b) Time to best response is shown for each of the 25 patients that had tumor reduction. Arrow on $\mathrm{x}$-axis indicates the first time point at which tumor response was assessed after start of therapy. Asterisks (*) indicate patients with ongoing response at last assessment. 
a

PFS

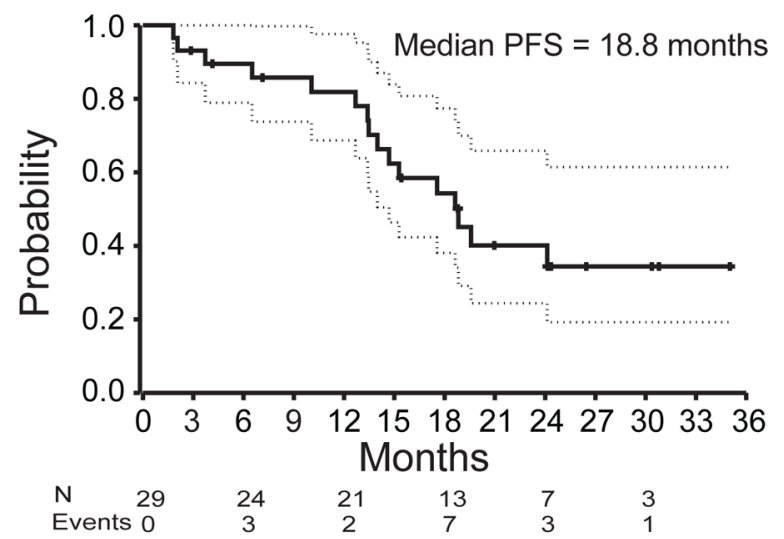

b $\quad$ FLIPI 1 PFS

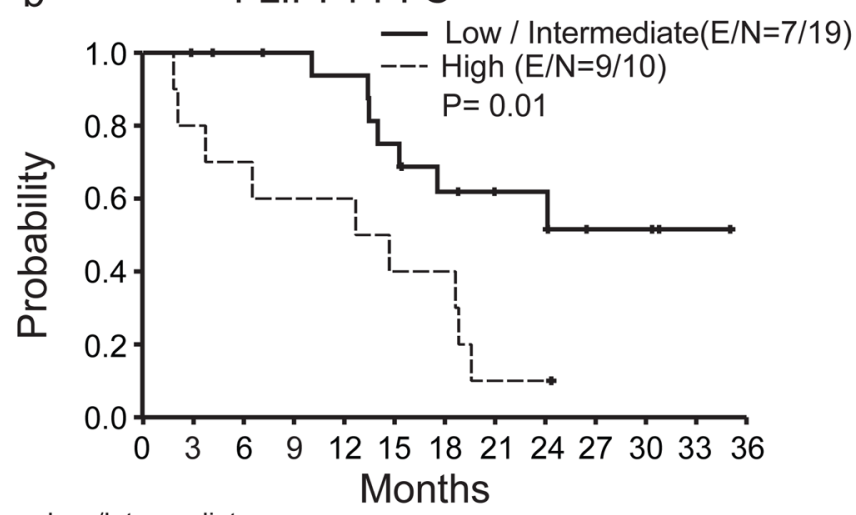

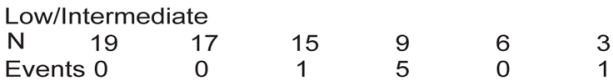

$\begin{array}{llllll}\text { High } & & & & & \\ \text { N } 10 & 7 & 6 & 4 & 1 & 0 \\ \text { Events } 0 & 3 & 1 & 2 & 3 & 0\end{array}$




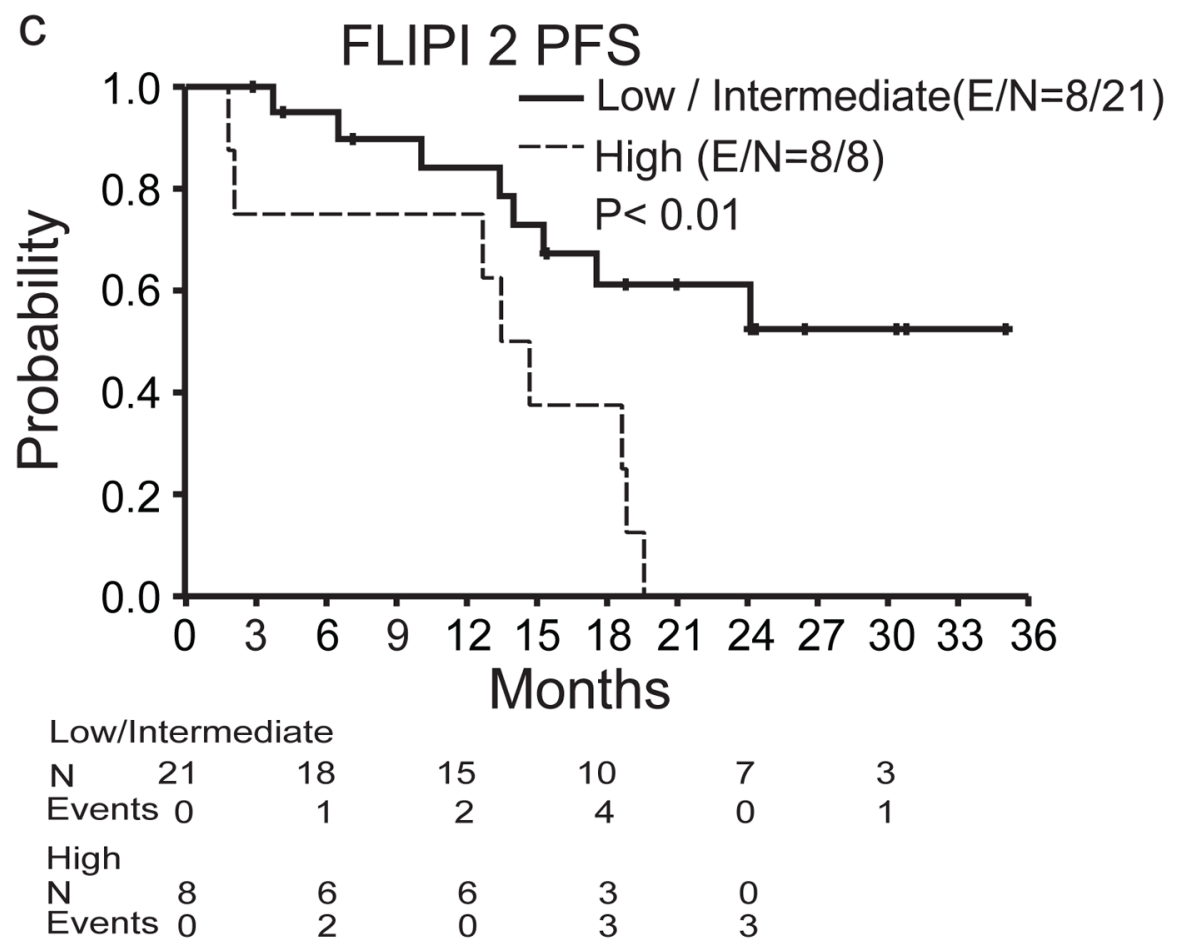

Figure 2. Progression-free survival (PFS) after pidilizumab and rituximab therapy a) Kaplan-Meier curve of PFS with $95 \%$ confidence intervals (CI). b and c) Kaplan-Meier curves of PFS for low/intermediate vs high risk groups for FLIPI 1 (b) and FLIPI 2 (c). The number of events $(\mathrm{E})$ and the total number of patients at risk $(\mathrm{N})$ over time and the $\mathrm{p}$ values by log-rank test are shown. 
a

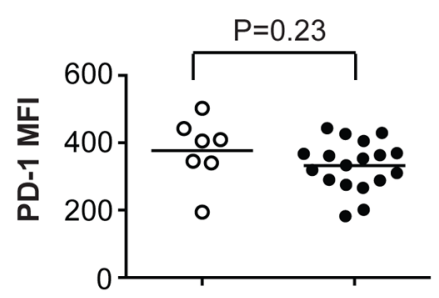

b

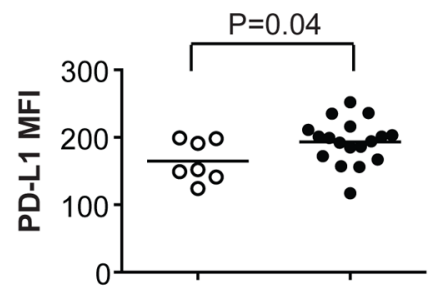

C

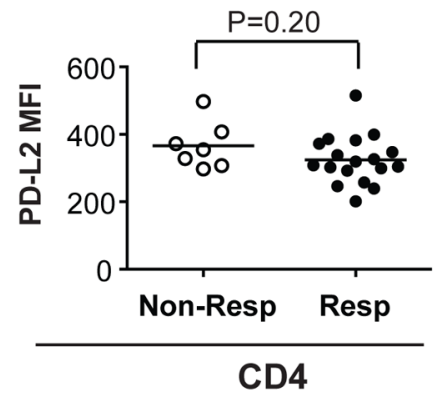

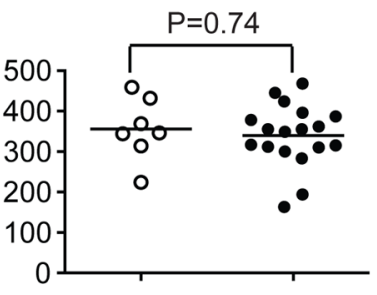
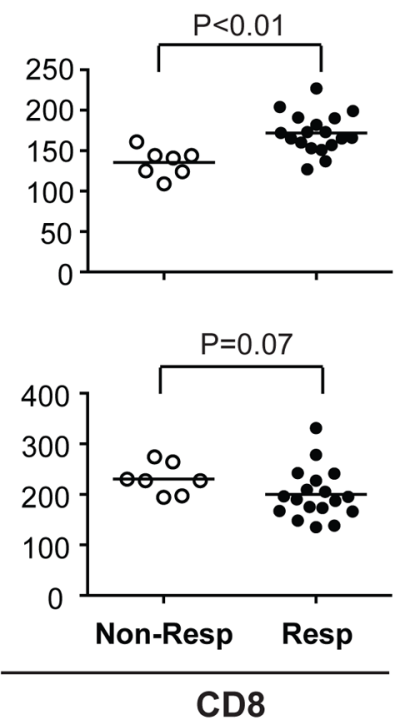
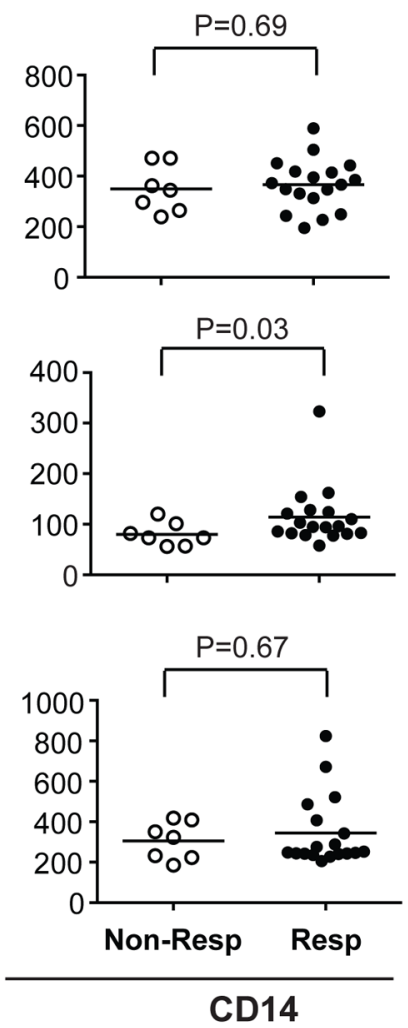

Figure 3. Expression of PD-1, PD-L1, and PD-L2 on peripheral blood T cells and monocytes a-c) The mean fluorescence intensity (MFI) of PD-1 (a), PD-L1 (b), and PD-L2 (c) on peripheral blood $\mathrm{CD}^{+}$and $\mathrm{CD}^{+} \mathrm{T}$ cells and monocytes was determined by flow cytometry on 25 patients (18 responders and 7 non-responders) with available PBMC samples at baseline. The horizontal line indicates the mean for each group. Wilcoxon rank-sum test was used to evaluate differences in marker expression between the two patient groups. 
Table 1

Patient Characteristics at Enrollment $(\mathrm{n}=30)$

\begin{tabular}{|llc|}
\hline Characteristic & & n (\%/Range) \\
\hline Age & Median (Range) & $61(35-79)$ \\
\hline FLIPI 1 & Male & $17(57)$ \\
& Female & $13(43)$ \\
& Low & $13(43)$ \\
& Intermediate & $7(23)$ \\
& High & $10(33)$ \\
\hline \multirow{2}{*}{ FLIPI 2 } & Low & $7(34)$ \\
& Intermediate & $15(50)$ \\
& High & $8(27)$ \\
\hline Prior Therapies & Median (Range) number of treatment regimens & $1(1-4)$ \\
& Median (Range) time (months) from last therapy & $23.8(9.8-76.1)$ \\
& Chemotherapy combination & $21(70)$ \\
& Biologic therapy combination & $11(37)$ \\
& Radioimmunotherapy & $2(7)$ \\
& Rituximab maintenance & $7(23)$ \\
& Rituximab monotherapy & $1(3)$ \\
& Any Rituximab & $30(100)$ \\
& Median (Range) number of prior rituximab doses & $7(2-22)$ \\
\hline
\end{tabular}


Table 2

Summary of Grades 1 and 2 Adverse Events $(\mathrm{n}=30)$

\begin{tabular}{|l|c|c|c|}
\hline Adverse Event & Grade 1, n (\%) & Grade 2, n (\%) & Grade 3/4, n \\
\hline Anemia & $14(47)$ & 0 & 0 \\
Fatigue & $13(43)$ & $2(7)$ & 0 \\
Leukopenia & $11(37)$ & 0 & 0 \\
Thrombocytopenia & $8(27)$ & $2(7)$ & 0 \\
Dyspnea & $6(20)$ & 0 & 0 \\
Neutropenia & $5(17)$ & $1(3)$ & 0 \\
Nausea & $5(17)$ & 0 & 0 \\
Sweating & $4(13)$ & 0 & 0 \\
Neuropathy & $4(13)$ & 0 & 0 \\
Cough & $4(13)$ & 0 & 0 \\
Pain & $3(10)$ & $2(7)$ & 0 \\
Edema & $3(10)$ & $1(3)$ & 0 \\
Pruritus & $3(10)$ & 0 & 0 \\
Diarrhea & $3(10)$ & 0 & 0 \\
Anorexia & $3(10)$ & 0 & 0 \\
Hypotension & $2(7)$ & $1(3)$ & 0 \\
Respiratory Infection & $1(3)$ & $5(17)$ & 0 \\
\hline
\end{tabular}




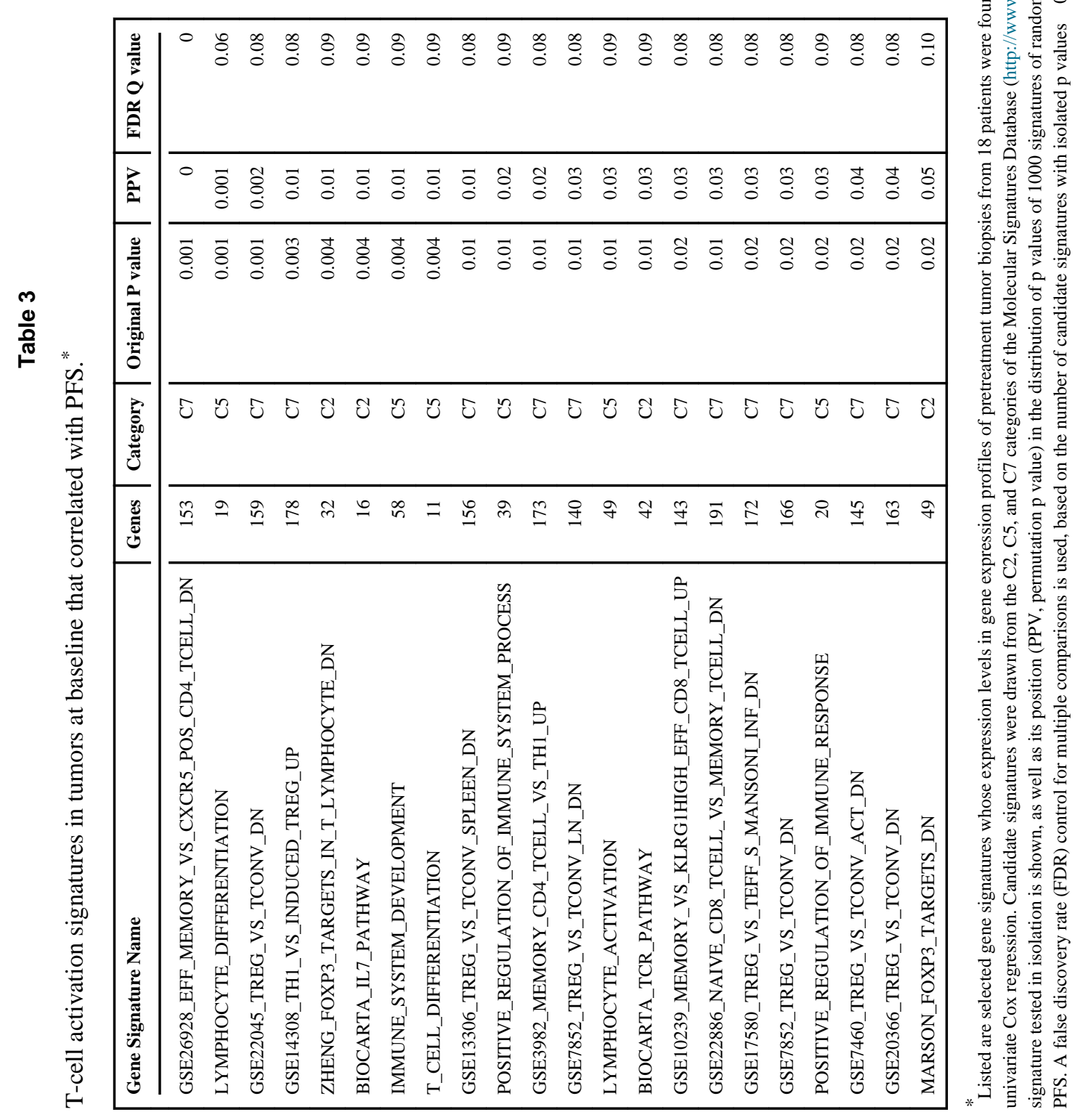




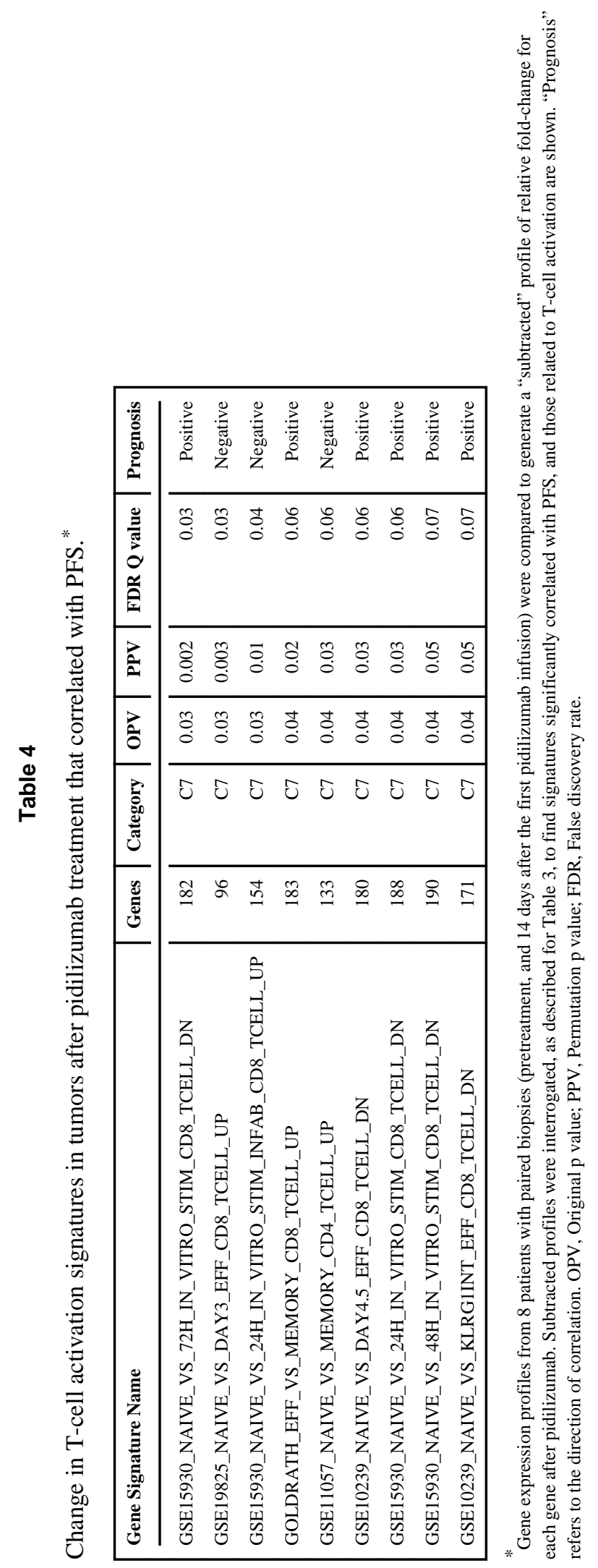

